\title{
Economía colaborativa y regulación: un análisis prospectivo
}

\author{
Collaborative economy and regulation: \\ a prospective analysis
}

\author{
José Manuel Ordóñez de Haro \\ José Luis Torres \\ Universidad de Málaga
}

\begin{abstract}
Resumen
La denominada economía colaborativa ha experimentado un intenso proceso de expansión durante las últimas décadas, principalmente en lo que respecta a determinados servicios en el sector turístico y el transporte. Aunque este fenómeno no es nuevo, ya que históricamente ha tenido una gran importancia en las economías anteriores a la revolución industrial, su resurgimiento en los últimos años está asociado a factores tecnológicos y a la acumulación de capital doméstico en forma de bienes duraderos. El progreso tecnológico y el desarrollo de las tecnologías de la información y la comunicación (TIC) han posibilitado la eliminación de determinadas barreras de mercado y limitaciones de información, permitiendo a los hogares dedicar recursos de la producción doméstica a la producción de bienes y servicios que son ofrecidos a otros consumidores. En este trabajo proponemos un marco teórico en el que estudiar el sector de la economía colaborativa, junto con el sector productivo de mercado y el sector de producción doméstica en el hogar. Estos tres sectores presentan características diferenciadas en términos de los factores productivos que se utilizan en cada uno de ellos, definiéndose la economía colaborativa como un sector intermedio entre la producción tradicional de mercado y la producción doméstica. Sobre la base de esta propuesta, se identifican una serie de problemas que se plantean en la regulación de la economía colaborativa, así como algunas consideraciones respecto a los servicios turísticos y de transporte en este contexto.

Palabras clave: economía colaborativa, producción doméstica, producción de mercado, regulación, turismo, transporte.
\end{abstract}

Clasificación JEL: D13, D16, L81, K2, L5.

\begin{abstract}
The so-called collaborative economy has experienced an intensive process of expansion during the last decades, mainly regarding certain tourism and transport services. Though this phenomenon is not new, since it played an important role in the economies prior to industrial revolution, its renaissance in recent years is associated with technological factors and the

* José Manuel Ordóñez de Haro desea agradecer la financiación recibida a través de la Cátedra de Política de Competencia de la Universidad de Málaga. Asimismo, José Luis Torres también desea agradecer la financiación recibida del Proyecto SEJ-122. Las opiniones expresadas por los autores son estrictamente personales y en ningún caso vinculantes para las instituciones en las que prestan sus servicios. Cualquier error u omisión es también exclusiva responsabilidad de los autores.
\end{abstract}

DOI: https://doi.org/10.32796/cice.2019.97.6798 
accumulation of domestic capital in the form of durable goods. Technological progress and the development of information and communication technologies (ICTS) have removed market barriers and information constraints, allowing households to devote home production inputs to the production of good and services that are provided to other consumers. In this paper we propose a theoretical framework within which to study the sector of the collaborative economy, together with the market production and household production sectors. These three sectors have distinct characteristics in terms of the productive factors that are used in each of them, with collaborative economy being defined as an intermediate sector between traditional market production and domestic production. On the basis of this proposal, we finally provide a review of a number of issues that arise in the regulation of collaborative economy, and some considerations applicable to tourism and transport services within this framework.

Keywords: collaborative economy, household production, market production, regulation, tourism, transport.

JEL classification: D13, D16, L81, K2, L5.

\section{Introducción}

En la última década hemos observado una significativa expansión en un amplio conjunto de actividades económicas que han dado lugar a la denominada economía colaborativa o consumo colaborativo. Se trata de un conjunto de actividades económicas que se realizan de forma paralela al mercado tradicional, principalmente en la prestación de servicios, y donde la oferta es realizada por los consumidores o familias, al igual que ocurre en el mercado de segunda mano, con el que comparte algunas características. Estas relaciones de consumo han sido posibles gracias al avance tecnológico y, en particular, a Internet, que ha permitido el desarrollo de una serie de servicios ofertados directamente por las familias y su acceso al mercado.

La importancia de este nuevo modelo de negocio ha hecho que la mayoría de economías modernas intenten cuantificar o, al menos, estimar su contribución a la producción total de las mismas (Coyle, 2018). En particular, la Comisión Europea ha estimado que la economía colaborativa generó en el año 2015 ingresos cercanos a los 4.000 millones de euros y facilitó transacciones dentro del territorio europeo por valor de 28.000 millones de euros (Vaughan y Daverio, 2016).

La expansión de las Tecnologías de la Información y la Comunicación (TIC) ha facilitado el que los hogares participen en nuevas clases de actividades online para las que, previamente, no había intermediarios del mercado, o si los había eran pocos y su capacidad para poner en contacto demandantes y oferentes muy limitada. El desarrollo de este sector ha provocado la aparición de nuevos actores en determinados mercados, lo que también ha generado ciertos problemas, debido a la inexistencia de un marco regulatorio previo. El interés académico por analizar este tipo de intercambios tiene su origen en las contribuciones realizadas por Botsman y Rogers (2010a, b) y Belk (2014), a los que han seguido un gran número de trabajos donde se abordan distintas cuestiones y problemáticas de este sector desde distintos enfoques.

El sector de la economía colaborativa presenta una serie de características particulares que lo hacen distinto al sector productivo tradicional. El concepto de economía 
colaborativa (collaborative economy) o economía compartida (sharing economy) ha generado bastante controversia respecto a las actividades que englobaría, en parte porque este término ha ido ampliando su espectro, abarcando inicialmente solo las actividades no comercializadas, estrictamente colaborativas, de persona a persona (peer-to-peer $)^{1}$, hasta incluir también las actividades comerciales ${ }^{2}$. Así, una primera definición tentativa, aunque bastante restrictiva, es la defendida por Frenken y Schor (2017) que consideran la economía colaborativa como aquella por la cual los consumidores se garantizan entre ellos el acceso temporal a determinados activos físicos que se encuentran infrautilizados, posiblemente a cambio de dinero. Frente a esta definición, existen otros autores que optan por acogerse a una definición más amplia que englobe los diferentes términos utilizados en esta materia, pero manteniendo una serie de elementos básicos presentes en todos ellos. Así, estas definiciones harían referencia a un nuevo modelo de negocio que se diferencia de los demás existentes, no por su naturaleza colaborativa sino por la existencia de intermediarios digitales con determinadas características que facilitan las transacciones ${ }^{3}$.

La Comisión Europea entiende el concepto de economía colaborativa como aquella referida a modelos de negocio en los que se facilitan actividades mediante plataformas colaborativas que crean un mercado abierto para el uso temporal de mercancías o servicios ofrecidos a menudo por particulares, no implicando estas transacciones, en general, un cambio de propiedad, y pudiendo ser con o sin ánimo de lucro (Comisión Europea, 2016a). En su definición, la Comisión caracteriza los agentes que se ven implicados en estos modelos distinguiendo entre prestadores de servicios, ocasionales («pares») o que actúan a título profesional; usuarios de dichos servicios; e intermediarios que, mediante plataformas online (plataformas colaborativas), conectan a prestadores y usuarios. En esta definición, por tanto, tendrían cabida tanto las personas privadas que comparten activos, recursos, tiempo y habilidades de manera ocasional, como prestadores de servicios en el ejercicio de su actividad profesional. Por ejemplo, en el sector turístico, esto tiene lugar cuando en las plataformas digitales operan como prestadores de servicios tanto las personas privadas, proporcionando acceso a bienes y recursos infrautilizados como sería ofrecer sus casas como alojamiento, sus coches como medios de transporte, o sus conocimientos a los visitantes, como empresas que ofertan las casas de su propiedad u otros recursos a los demandantes de sus servicios, permitiendo un acceso temporal a esos bienes, recursos o servicios (World Tourism Organization, 2017). Las plataformas digitales han permitido alcanzar mayores niveles de eficiencia en el emparejamiento de

\footnotetext{
1 Desde este punto de vista, ciertas actividades como las llevadas a cabo por Airbnb no pueden ser consideradas como economía colaborativa y presentan las características de una actividad comercial de mercado. Tampoco lo serían ciertos servicios realizados por Uber.

${ }^{2}$ Asimismo, Sutherland y Jarrahi (2018) identifican en la literatura diferentes enfoques e intereses, según el campo de investigación, en el análisis de este concepto y su relación con las plataformas digitales.

${ }^{3}$ De hecho, esta definición, en la que también se incorporan transacciones entre empresas y consumidores, estaría incluyendo relaciones que ya tienen o podrían tener lugar en el mercado tradicional.
} 
oferentes y demandantes de bienes y servicios, así como en el uso de determinados activos propiedad de los hogares (Coyle, 2018).

En este trabajo entendemos la economía colaborativa como una porción del mercado que usa factores productivos que forman parte del sector de producción en el hogar, que previamente no estaban comercializados y que permanecían sin ser utilizados por los propietarios de los mismos. Por tanto, en nuestra definición nos centramos en una de las características principales de este sector: una gran parte de los servicios que se comercian son generados por activos de capital doméstico que está formado por el gasto (o inversión) de los hogares en bienes duraderos. Además, la función de producción del sector de la economía colaborativa no solo sería una función de la dotación de capital o bienes duraderos puesta a disposición de otros consumidores, sino que también incluiría la dotación de tiempo (trabajo) que se asigna a estas actividades. Desde este punto de vista, el gasto en bienes duraderos por parte de las familias puede ser considerado como una inversión, que va a dar lugar a una dotación de capital en el hogar. Este capital tiene como objetivo fundamental ser utilizado en la producción doméstica, si bien también existe la posibilidad de que este capital se utilice, asimismo, para la producción de servicios vendidos en el mercado. Bienes típicos que pueden ser puestos en valor de mercado son los vehículos (equipos de transporte) y las viviendas (estructuras residenciales), denominados por Benkler (2004) como «bienes compartibles», aunque también pueden considerarse otros bienes duraderos.

En este artículo, pretendemos contextualizar el sector de la economía colaborativa, analizando sus características desde un punto de vista estrictamente económico, así como trazar algunos elementos que pueden determinar su evolución futura y su interacción con el resto de sectores de la economía. Para ello, presentamos un marco teórico básico que nos permite situar la economía colaborativa y su interrelación tanto con el sector productivo tradicional, como con la producción en el hogar, con los que comparte algunos elementos en común ${ }^{4}$. Este marco teórico permite determinar las principales características de este mercado y puede constituir la base teórica de partida para analizar diferentes efectos de la irrupción y desarrollo de estas actividades, así como para estudiar sus implicaciones de política económica.

El modelo desarrollado considera tres sectores productivos: el sector productivo tradicional, la producción doméstica y el sector de economía colaborativa. El análisis de la producción de bienes y servicios domésticos ha sido realizado por numerosos autores, como una producción alternativa a la del mercado. Benhabid, Rogerson y Wright (1991) han desarrollado un modelo de ciclo real para estudiar las implicaciones de la producción doméstica y su interacción con el sector productivo. Greenwood y Hercowitz (1991) estudian la asignación de tiempo y de capital entre actividades de mercado y actividades domésticas para la economía norteamericana.

\footnotetext{
${ }^{4}$ Los estudios teóricos que analizan las implicaciones de la economía colaborativa sobre el bienestar y sobre el sector productivo tradicional son relativamente escasos y recientes. Cabe destacar, por ejemplo, Einav et al. (2016), Weber (2016) o Gal-Or (2018).
} 
Greenwood, Seshadri y Yorukoglu (2005) apuntan a que la electricidad dio lugar a un gran número de bienes duraderos que supusieron una revolución en las economías domésticas, siendo uno de los factores fundamentales en la liberación de la mujer y su incorporación al mercado de trabajo.

En este contexto teórico, la economía colaborativa surge cuando las familias utilizan su dotación de factores productivos para ponerlos a disposición del mercado, ya se trate de capital o de trabajo. En este sentido, no se diferenciaría del resto de actividades productivas de mercado existentes, ya que consiste en un alquiler de factores productivos. Sin embargo, se diferencia en el hecho de que pone a disposición del mercado factores productivos que inicialmente solo se utilizan en la producción de bienes y servicios domésticos, esto es, factores productivos que previamente no se comercializaban. Por tanto, se trataría de un sector que comparte características tanto del sector productivo de la economía (por los bienes y servicios que ofrece) como del sector de producción doméstica (por los factores productivos que utiliza).

Uno de los elementos claves de la economía colaborativa es que aumenta la cantidad de capital disponible en la economía. Así, las familias cuentan con una dotación de capital que, en términos relativos, es muy elevada, que podría cuantificarse a través del consumo en bienes duraderos. Esta dotación de capital resulta, en muchos casos, infrautilizada (Frenken y Shor, 2017), por lo que podría existir una determinada rentabilidad para poner en el mercado dicha capacidad productiva no utilizada. Las economías domésticas disponen de una dotación de capital, que en principio va destinada a la producción de bienes y servicios domésticos. Este capital está compuesto fundamentalmente por bienes de consumo duraderos, que tienen la forma bien de estructuras (como la vivienda) o equipos (como los vehículos), y que por tanto, son susceptibles de ser usados en la producción de bienes y servicios tanto domésticos como de mercado.

La economía colaborativa hace que el gasto en consumo en bienes duraderos se pueda transformar en un gasto de inversión, y que los bienes duraderos, incluyendo las estructuras residenciales, puedan considerarse como capital productivo de la economía. Teniendo en cuenta la competencia que se produce entre el sector productivo tradicional y el sector de la economía colaborativa, resulta de interés conocer las consecuencias de los posibles escenarios en los que esta competencia tiene lugar, así como sus implicaciones económicas para los diferentes actores económicos participantes. Nuestra modelización también constituye un primer paso para poder evaluar la aportación del sector colaborativo al bienestar de consumidores, familias, empresas, y de la economía en su conjunto.

Asimismo, en un contexto donde las perturbaciones que genera la competencia de los operadores de la economía colaborativa sobre los operadores del sector productivo tradicional parecen justificar la intervención de las autoridades para introducir medidas reguladoras del sector colaborativo, nuestro modelo teórico ofrece la posibilidad de contemplar los efectos que tendrían dichas medidas, y si realmente conllevan una mejora del bienestar económico global. 
La estructura del resto del artículo es como sigue. En la sección segunda realizamos una breve revisión de las razones que subyacen a la evolución y rápido crecimiento de la economía colaborativa. La tercera sección plantea una serie de reflexiones sobre la relación entre el mercado productivo tradicional, las economías domésticas y la economía colaborativa. El modelo teórico que proponemos para la representación de la economía colaborativa como un tercer sector de la economía se desarrolla en la sección cuarta. La quinta sección aborda las principales tesis y cuestiones a la hora de considerar la regulación aplicable a la economía colaborativa. La sección sexta contiene las principales conclusiones del trabajo.

\section{Del consumidor al «prosumidor»}

La economía colaborativa surge cuando el consumidor se transforma en lo que se ha venido a llamar «prosumidor». Aunque este último concepto no se encuentra siempre bien delimitado, el «prosumidor» haría referencia a un consumidor que actúa como productor ${ }^{5}$. Esta función de los consumidores no es nueva, sino que dentro del hogar, los miembros de la familia realizan una gran variedad de actividades productivas, que no forman parte del mercado. Así, parte de los bienes o servicios que consume un individuo los produce él mismo, utilizando unos factores productivos propios, frente a aquellos bienes y servicios que se pueden comprar en el mercado.

Si pensamos en una economía primitiva, poco desarrollada, la mayoría de los productos y servicios proceden del sector primario, así como de una industria manufacturera básica. En este contexto, hemos de pensar que un gran número de transacciones se realizan dentro de lo que hoy definimos como economía colaborativa. En estas economías poco desarrolladas, la economía colaborativa representa un porcentaje relativamente elevado de la actividad económica, por cuanto la mayoría de transacciones corresponden a producción doméstica que se comercia directamente en el mercado. El caso extremo sería una economía de intercambio puro o de trueque, donde simplemente se cambia un bien por otro, si bien en el estado actual la economía colaborativa se centra fundamentalmente en el comercio de servicios, estando muy limitado el correspondiente a bienes que se corresponderían con producción doméstica.

El Gráfico 1 muestra la evolución de la economía colaborativa en función del estado de desarrollo de la economía. Partiendo de un estado de desarrollo bajo, la economía colaborativa representaría un elevado número, en términos relativos, del total de transacciones que se llevan a cabo. A medida que la economía se desarrolla y adquieren peso los sectores manufactureros con tareas más mecanizadas, el peso relativo de la economía colaborativa va disminuyendo, alcanzando su mínimo en las fases de mayor desarrollo industrial. Sin embargo, el progreso tecnológico hace que

${ }^{5}$ En otros contextos, el concepto haría referencia a consumidores que llegan a ser semiprofesionales en el conocimiento y uso de determinados bienes, gracias a la mayor información y formación que está a su alcance. 


\section{GRÁFICO 1 \\ ECONOMÍA COLABORATIVA Y DESARROLLO ECONÓMICO}

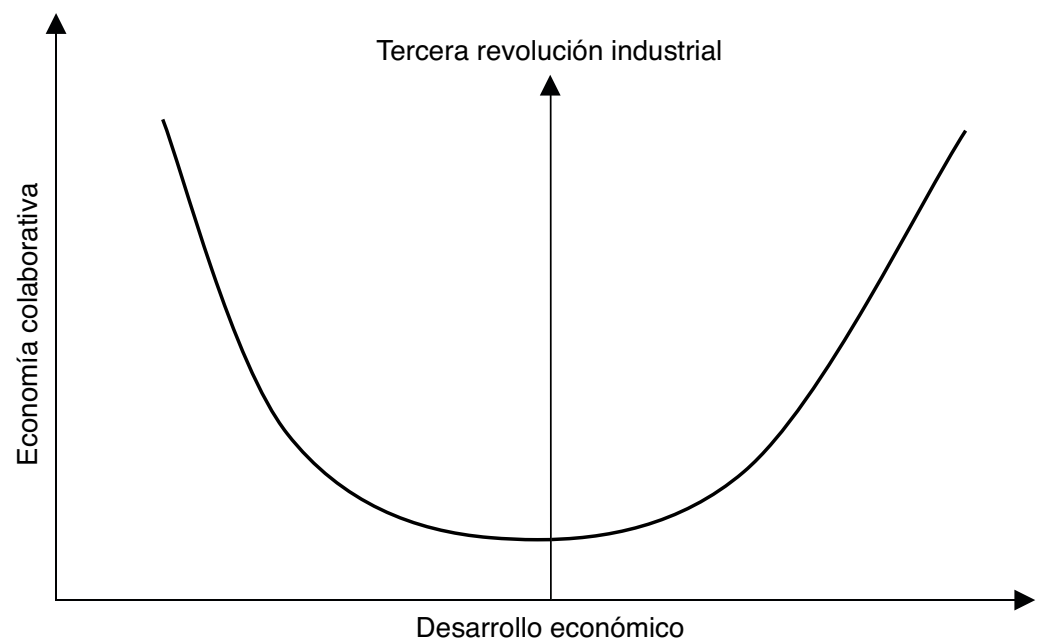

FUENTE: Elaboración propia.

a partir de un determinado nivel de desarrollo, lo que hemos denominado tercera revolución industrial, en la cual los servicios adquieren una gran importancia, el peso relativo de la economía colaborativa vuelva a aumentar. Esta sería la situación en la que se encuentran las economías desarrolladas en la actualidad, lo que nos llevaría a un desarrollo progresivo de la economía colaborativa en el futuro.

El resurgimiento de la economía colaborativa en los últimos años puede explicarse por una multitud de factores, ligados al desarrollo del sector servicios y al progreso tecnológico. Uno de los elementos clave de este desarrollo ha sido Internet y las plataformas desarrolladas para la oferta de determinados servicios, tales como Uber o Zipcar para el transporte, Airbnb para los alojamientos, TaskRabbit para compartir trabajo, EatWith para la preparación de comidas, entre otras (véase Cuadro 1). En un estudio encargado por la Comisión Europea, centrado en nueve Estados miembros de la Unión Europea ${ }^{6}$, se estimaba que, hasta el año 2016, se habían creado al menos 275 plataformas de economía colaborativa (Vaughan y Daverio, 2016).

Este elemento tecnológico ha supuesto la eliminación de barreras de entrada y segmentación de mercados, constituyendo medios de publicidad y de información de ofertas por parte de consumidores que previamente no tenían esta posibilidad. De hecho, en otro estudio realizado para la Comisión Europea, basado en una encuesta con 14.050 entrevistas llevadas a cabo en los 28 Estados miembros de la Unión Europea en marzo de 2016, el 17 por 100 de los encuestados contestó que habían utilizado Bajos.

${ }^{6}$ Concretamente en Francia, Bélgica, Alemania, Reino Unido, Polonia, España, Italia, Suecia y Países 
en alguna ocasión las plataformas colaborativas, y de estos más del 30 por 100 las habían usado para prestar servicios (Comisión Europea, 2016b).

Los problemas de información asimétrica entre productores y consumidores han sido y son habitualmente planteados como la justificación para introducir medidas reguladoras que protejan a los consumidores frente a los riesgos de estas relaciones comerciales. Así, en los primeros momentos, estas transacciones de consumidores presentaban riesgos elevados, por cuanto cada una de las partes tenía una información muy limitada de la otra, y se producía un problema de confianza o fiabilidad que incluso desincentivaba la participación de los consumidores en transacciones electrónicas.

Sin embargo, los avances en Internet, proporcionando potentes herramientas de búsqueda y vigilancia, así como mecanismos reputacionales de retroalimentación han venido a solucionar o, como mínimo, reducir los problemas de información asimétrica. Estos mecanismos pueden clasificarse en términos generales en mecanismos centralizados o de terceros, donde es una tercera parte la que, además de conectar a compradores y vendedores, busca añadir confianza y validez a las transacciones, y mecanismos entre pares que directamente aumentan la confianza entre las dos partes implicadas en la transacción (Thierer et al., 2016).

El desarrollo de plataformas digitales que integran estos mecanismos de reputación ha supuesto la reducción sustancial de los riesgos originalmente asociados a las transacciones online, lo que ha posibilitado el rápido desarrollo de la economía colaborativa. De hecho, la economía colaborativa se puede interpretar como un caso especial de un mercado de plataformas o bilateral, en el que se emplea la tecnología para poner en contacto gran número de compradores y vendedores. Los mercados bilaterales surgen cuando dos tipos diferentes de usuarios pueden beneficiarse de la interacción que se produce entre ellos a través de una o más plataformas o intermediarios $^{7}$. Las plataformas que operan en la economía colaborativa obtienen, generalmente, sus ingresos en forma de comisiones que cobran a todos o a una parte de los participantes en las transacciones.

Hasta la actualidad, la economía colaborativa ha tenido una mayor expansión en una serie de sectores específicos. Como se aprecia en el Cuadro 1, uno de esos sectores en los que ha tenido mayor repercusión esta nueva forma de negocio ha sido el sector turístico. Tal y como indican Dredge y Gyimóthy (2015), la economía colaborativa, para el caso del sector turístico, engloba a un amplio conjunto de actividades, permitiendo a turistas y residentes compartir sus hogares, coches, comidas, y sus conocimientos del medio local actuando, por ejemplo, como guías turísticos.

Según Cheng (2016), una multitud de factores han impulsado la colaboración o acceso compartido en el sector del turismo: los turistas buscan una mejor calidad-precio, productos de turismo sostenible y experiencias turísticas más auténticas. La economía colaborativa, desde el punto de vista de la oferta, ha ampliado la oferta

\footnotetext{
${ }^{7}$ Véase, por ejemplo, Rochet y Tirole (2006) y Evans y Schmalensee (2015).
} 


\section{CUADRO 1}

PRINCIPALES SECTORES ECONÓMICOS CON PRESENCIA DE LA ECONOMÍA COLABORATIVA

\begin{tabular}{|c|c|c|}
\hline Sector de actividad & $\begin{array}{l}\text { Ejemplos de plataformas } \\
\text { digitales intermediarias }\end{array}$ & Descripción orientativa \\
\hline Transporte & $\begin{array}{c}\text { Uber, Lyft, BlaBlaCar, Rdvouz, } \\
\text { Click\&Boat, GetAround, } \\
\text { Zipcar, Avancar. }\end{array}$ & $\begin{array}{l}\text { Contratación de servicios de } \\
\text { transporte de pasajeros para } \\
\text { trayectos tanto de corta como } \\
\text { larga distancia, así como viajes } \\
\text { compartidos por pasajeros, me- } \\
\text { diante la puesta en común de un } \\
\text { vehículo privado que realiza ese } \\
\text { trayecto. }\end{array}$ \\
\hline Alojamiento & $\begin{array}{c}\text { Airbnb, HomeAway, } \\
\text { Couchsurfing, LoveHomeSwap, } \\
\text { HomeExchange, Niumba. }\end{array}$ & $\begin{array}{l}\text { Transacciones para el hospe- } \\
\text { daje en camas, habitaciones, } \\
\text { apartamentos, casas, mediante } \\
\text { alquiler o intercambio de casas } \\
\text { en períodos de corta duración o } \\
\text { vacacionales. }\end{array}$ \\
\hline Alimentación & $\begin{array}{l}\text { EatWith, Feastly, VizEat, } \\
\text { ShareTheMeal, ShareFood. }\end{array}$ & $\begin{array}{l}\text { Transacciones entre individuos } \\
\text { para la prestación de servicios } \\
\text { de comidas y bebidas comparti- } \\
\text { das en un entorno privado. }\end{array}$ \\
\hline Otras actividades turísticas & $\begin{array}{l}\text { ToursByLocals, BeMyGuest, } \\
\text { Vayable, GuruWalk. }\end{array}$ & $\begin{array}{l}\text { Transacciones entre demandan- } \\
\text { tes de rutas, excursiones, atrac- } \\
\text { ciones y otras actividades turís- } \\
\text { ticas guiadas y/u organizadas } \\
\text { por personas del lugar. }\end{array}$ \\
\hline Otros servicios & $\begin{array}{c}\text { TaskRabbit, WeTasker, Upwork, } \\
\text { HolterWatkin, Geniuzz. }\end{array}$ & $\begin{array}{l}\text { Transacciones entre deman- } \\
\text { dantes de servicios domésticos } \\
\text { o profesionales y proveedores } \\
\text { autónomos que tienen esa ca- } \\
\text { pacidad o habilidades infrauti- } \\
\text { lizadas. }\end{array}$ \\
\hline Servicios de financiación & $\begin{array}{c}\text { FundingCircle, } \\
\text { SyndicateRoom, LendingClub, } \\
\text { Kickstarter, MyNbest. }\end{array}$ & $\begin{array}{l}\text { Financiación colaborativa por } \\
\text { la que se ponen en contacto per- } \\
\text { sonas y empresas para prestar } \\
\text { o pedir prestado dinero entre } \\
\text { ellos, como forma alternativa } \\
\text { a la intermediación tradicional } \\
\text { realizada, por ejemplo, por los } \\
\text { bancos. }\end{array}$ \\
\hline
\end{tabular}

FUENTE: Elaboración propia basada en World Tourism Organization (2017) y Vaughan y Daverio (2016). 
global de opciones de turismo, facilitado el acceso de nuevos oferentes al abaratar el coste de crear un negocio turístico, así como de los consumidores a un amplio conjunto de productos y servicios mucho más asequibles que los ofrecidos por el sector turístico tradicional. Altinay y Taheri (2018) destacan que el crecimiento de la economía colaborativa en el sector turístico tiene que ver también con la forma en la que los consumidores con alto poder adquisitivo prefieren compartir y comunicarse con residentes locales para disfrutar de la cultura y tradiciones del país anfitrión, y mejorar su experiencia personal del viaje.

Otro de los sectores en el que este tipo de comercio se ha desarrollado significativamente es el de transporte (Botsman y Roger, 2010b; Bardhi y Eckhardt, 2012). También en este caso el fenómeno de compartir un vehículo para realizar desplazamientos no es nuevo, sin embargo, lo que han conseguido las nuevas innovaciones ha sido impulsar un cambio de actitud de particulares o empresas para permitir el acceso a vehículos de su propiedad (coches, bicicletas, etc.) a personas ajenas, para que hagan un uso temporal de los mismos, o para que ocupen una plaza en los mismos en los desplazamientos que realicen. Resulta importante destacar que la mayoría de estos nuevos modelos de movilidad han tenido una mayor implantación en ciudades con mayor densidad de población donde las oportunidades comerciales, así como los problemas de congestión de tráfico y de aparcamiento son más importantes, y donde han encontrado un rechazo total por parte de los prestadores privados de servicios de transporte pertenecientes a modelos tradicionales de movilidad (taxis), que consideran a la economía colaborativa una grave amenaza para la subsistencia de su modelo de negocio, al menos en su formato actual.

El hecho de que la economía colaborativa haya avanzado más en ciertos sectores vinculados con la prestación de determinados servicios, no significa que este proceso de expansión se vaya a limitar a los mismos. Es de esperar que, a medida que se produzcan mayores avances tecnológicos, se incorporen a este sector nuevos bienes y servicios.

\section{Economía colaborativa: progreso tecnológico y bienes duraderos}

Tal y como hemos indicado anteriormente, la economía colaborativa comparte características de la economía productiva de mercado y de la economía doméstica. En este apartado, vamos a analizar un conjunto de características fundamentales que definen a la economía colaborativa. Con este fin, podemos distinguir la existencia de tres sectores productivos con características diferenciadoras.

En primer lugar, la producción de mercado, que emplea tanto trabajo como capital físico en forma de estructuras comerciales y maquinaria y equipos que tienen como destino la producción de bienes y servicios de mercado.

En segundo lugar, la economía doméstica, en la cual se usa un tiempo distinto al de trabajo, pero que no podemos considerar como ocio, y capital doméstico, que es distinto al capital productivo de mercado. Este capital doméstico está compuesto 
por las estructuras domésticas (viviendas), y por bienes duraderos que constituyen equipos (equipos de transporte en el caso de los vehículos y otros equipos usados para la producción de bienes y servicios domésticos, tales como neveras, hornos, microondas, etc.).

En tercer lugar, la economía colaborativa, que produce bienes y servicios de mercado, pero utilizando los activos de capital doméstico y, en determinados casos, también tiempo de trabajo adicional al de mercado ${ }^{8}$. Esto hace que la economía colaborativa se sitúe a medio camino entre la producción de mercado y la producción doméstica. Dos son los elementos que definen la expansión de la economía colaborativa: la eliminación de barreras de entrada y problemas de información gracias al progreso tecnológico, y la existencia de un capital doméstico compuesto por bienes duraderos que pueden ser compartidos.

\section{Progreso tecnológico, barreras de entrada e información}

Uno de los factores fundamentales que ha permitido el importante desarrollo que ha experimentado recientemente la economía colaborativa es, sin duda alguna, el progreso tecnológico, principalmente el relacionado con las tecnologías de la información y de la comunicación (TIC). Estas tecnologías han supuesto la eliminación de las barreras de entrada existentes en este tipo de mercado y el acceso a un mercado global. Así, por ejemplo, las viviendas han existido siempre, lo mismo que el mercado de alquileres. Sin embargo, la vivienda con fines turísticos ha tenido tradicionalmente un desarrollo más limitado, debido a las restricciones de información para poner en contacto a demandantes y oferentes de este servicio. Esta dificultad de acceso a este servicio había limitado en gran medida su desarrollo como servicio turístico de corta duración en el tiempo. Sin embargo, la irrupción de Internet y de aplicaciones específicas para este tipo de mercado, han permitido su desarrollo, especialmente importante en algunos destinos, y provocado diferentes externalidades negativas.

Estas externalidades se refieren a aquellas circunstancias en las que actividades productivas o de consumo realizadas en el contexto de la economía colaborativa afectan negativamente a agentes distintos a los que han generado esos efectos. Más concretamente, en la actividad de alojamiento de corta duración, las externalidades más evidentes provienen de los efectos adversos que provocan los inquilinos de estos alojamientos sobre el vecindario (ruido, sobresaturación de espacios públicos, daños en los recursos comunes, entre otros), así como la disminución drástica de inmuebles en el mercado del alquiler de larga duración o la subida de los precios de los alquileres, ya que la mayoría de los propietarios de esos inmuebles preferirían ahora la rentabilidad que les ofrece el alojamiento de corta duración en el marco de la economía colaborativa. También, en el sector del transporte, este nuevo modelo

${ }^{8}$ Un sector particular que tiene características similares al sector de la economía colaborativa es el de segunda mano. 
de negocio genera externalidades que tienen que ver con los potenciales daños tanto para clientes como para no clientes, provocados por la falta de seguridad que tendrían determinados vehículos y/o sus conductores, incluyendo la que se refiere al nivel de cobertura del seguro de responsabilidad que poseen para responder por los potenciales daños que pudieran causar con su actividad (Edelman y Geradin, 2016).

Precisamente, estas externalidades a las que nos hemos referido suelen aducirse para justificar la intervención reguladora en estos nuevos mercados. No obstante, como veremos en la sección quinta del trabajo, a la justificación de la necesidad de esa regulación debería acompañar, en todo caso, la aplicación del principio de proporcionalidad y eficacia para evitar una regulación que conduzca a soluciones aún más ineficientes.

\section{Bienes duraderos y bienes compartibles}

Otro de los elementos clave a la hora de analizar el sector de la economía colaborativa es el referente a la acumulación de capital doméstico. Parte de los bienes y servicios que consumen las personas, los producen esas mismas personas en sus hogares. A esta parte de la producción es a la que denominamos producción doméstica. Se trata de bienes y servicios no de mercado, que no están destinados a la venta y que suponen un autoconsumo. Para producir estos bienes y servicios en el hogar, los individuos utilizan parte de su tiempo disponible y el stock de capital disponible en estos hogares, que está constituido por el consumo en bienes duraderos. Desde este punto de vista, el gasto en bienes duraderos puede considerarse como un gasto en inversión de activos de capital domésticos, que van desde la nevera, los vehículos, el horno, el lavavajillas, la lavadora, hasta la propia vivienda.

La dotación de bienes duraderos disponible por las familias tiene un valor de mercado. De hecho, estos bienes duraderos pueden calificarse como bienes compartibles, ya que pueden generar unos servicios que no tienen que ser obligatoriamente consumidos por los integrantes del hogar, sino que también pueden ser utilizados por otros consumidores ajenos al mismo. El problema es cómo hacer que estén disponibles los servicios que se pueden generar con estos bienes duraderos de tal manera que sean rentables, siendo la información la principal barrera existente para el desarrollo de estas actividades.

\section{Economía colaborativa: un marco teórico}

A pesar de la importancia que está adquiriendo la economía colaborativa, no existe un marco teórico que permita estudiar sus implicaciones sobre la economía a nivel agregado y sus interrelaciones con el resto de sectores. En la literatura existen diferentes análisis empíricos, concentrados en determinadas actividades que han experimentado un desarrollo muy elevado, tales como el de las viviendas turísticas, sector 
que ha recibido una gran atención, tanto por la academia como a nivel general, y que ha supuesto importantes cambios tanto para el sector hotelero tradicional (con el que compite de forma directa), como para el desarrollo de las ciudades y la convivencia en las mismas. Sin embargo, el fenómeno de la economía colaborativa va más allá de estos sectores particulares, en los que su expansión ha sido muy significativa, y es de prever que los continuos avances tecnológicos lleven a su aparición y consolidación en otros sectores productivos, incluyendo el manufacturero.

Tal y como hemos indicado anteriormente, una de las características fundamentales de la economía colaborativa es poner en el mercado un capital, formado principalmente por bienes duraderos y estructuras residenciales, propiedad de las familias, para la producción de determinados bienes y servicios que se comercian en el mercado. Estas dotaciones de capital, por lo general se encuentran infrautilizadas (por ejemplo, ciertos estudios muestran que los vehículos solo son utilizados alrededor de un 3-4 por 100 del tiempo, estando estacionados y sin uso el tiempo restante, 97-96 por 100$)^{9}$.

El marco teórico que proponemos consiste en definir la economía como tres sectores: el sector de mercado, el sector de producción doméstica y el sector de economía colaborativa. El análisis de la producción doméstica fue iniciado por Becker (1965) y Gronau (1973a, b), los cuales insisten en la importancia que tienen las actividades productivas realizadas dentro del hogar, tales como cocinar, limpiar, lavar la ropa, el cuidado de los hijos, entre otras. Así, el hogar se puede considerar como una unidad de producción que combina inputs de trabajo y activos de capital doméstico (vivienda, vehículos, herramientas, electrodomésticos), y compra bienes y servicios intermedios o finales, para producir una amplia gama de bienes y servicios finales que generalmente destina a su propio consumo, pero que también pueden llegar a poner en el mercado.

En la literatura existen diferentes desarrollos que han estudiado la interacción entre el mercado y el sector de producción doméstica. Ejemplos destacados de estos trabajos son Benhabid, Rogerson y Wright (1991), Greenwood y Hercowitz (1991), y McGrattan, Rogerson y Wright (1997). Estos autores han analizado la importancia de la producción doméstica, en términos de producción y de cantidad de factores productivos (tiempo y bienes duraderos), así como su interrelación con las actividades de mercado. En este contexto teórico, las familias toman dediciones de cuánto tiempo dedicar a trabajar en el mercado y cuánto tiempo dedicar a la realización de tareas domésticas. Al mismo tiempo, las familias deciden cuánto ahorran e invierten en capital de mercado y cuántos recursos destinan a la adquisición de bienes duraderos, que conformarían el stock de capital del sector doméstico, así como cuánto tiempo dedican a la producción de bienes y servicios domésticos. Tanto el capital doméstico como su trabajo serían susceptibles de utilizarse para ser comercializados en el sector de la economía colaborativa.

\footnotetext{
9 Véase Bates y Liebling (2012).
} 


\section{Las familias}

El consumo de las familias estaría compuesto por tres tipos de bienes y servicios: bienes y servicios de mercado que se compran a las empresas; bienes y servicios domésticos que son producidos en el hogar; y bienes y servicios compartidos adquiridos a otros consumidores. Estos tres tipos de bienes y servicios no son homogéneos, es decir, no son perfectamente sustitutivos. Si suponemos que la función de utilidad de las familias depende únicamente del consumo y del ocio, su función de utilidad la podemos definir como:

$$
U=U\left(C_{m, t}, C_{h, t}, C_{s, t} ; O_{t}\right)
$$

Donde $C_{m, t}$ es el consumo de bienes y servicios producidos por el mercado, $C_{h, t}$ es la cantidad de bienes y servicios de producción doméstica, $C_{s, t}$ es la cantidad de bienes y servicios adquiridos en el sector de economía colaborativa y $O_{t}$ es el tiempo dedicado al ocio por parte de las familias.

Los hogares pueden ahorrar en la forma de dos tipos de activos de capital: activos de capital físico que van a ser utilizados por las empresas para producir, o bien pueden ahorrar a través de la compra de bienes duraderos. Si bien tradicionalmente la compra de bienes duraderos se ha considerado parte del consumo, el desarrollo de los mercados de segunda mano y de la economía colaborativa hace que estos bienes puedan ser considerados en nuestro contexto como un activo de capital y, por tanto, forman parte del proceso de inversión. En este sentido, un automóvil es considerado tradicionalmente como un activo de capital si lo adquiere una empresa, o bien como un consumo de bien duradero si lo adquiere un particular. Desde el momento en que existe la posibilidad de que el particular utilice dicho vehículo para actividades comerciales (comparta su coche con otros usuarios a cambio de un ingreso monetario), ya tendríamos que tratarlo como un activo de capital que formaría parte de la inversión. Por tanto, las familias pueden invertir en capital de mercado o en capital doméstico, y este último puede ser utilizado tanto para la producción de bienes y servicios en el hogar, como para la producción de bienes y servicios en el sector de la economía colaborativa. Por tanto, la restricción presupuestaria a la que se enfrenta la familia podríamos definirla como:

$$
C_{m, t}+C_{h, t}+C_{s, t}+I_{k, t}+I_{h, t}=W_{m, t} L_{m, t}+W_{s, t} L_{s, t}+R_{t} K_{t}+R_{t}^{s} H_{t}
$$

Donde $I_{k, t}$ sería la inversión en activos de capital de mercado, $I_{h, t}$ es la compra de bienes duraderos, que constituiría la inversión en activos de capital doméstico, $W_{m, t}$ sería el salario en el mercado productivo tradicional, $L_{m, t}$ el empleo (fracción de tiempo dedicada a trabajar en el mercado), $W_{s, t}$ sería la retribución al trabajo en la producción colaborativa ${ }^{10}, L_{s, t}$ el tiempo dedicado a la actividades productivas en la economía colaborativa, $R_{t}$ la rentabilidad de los activos de capital de mercado, $K_{t}$ el

\footnotetext{
${ }^{10}$ Esta retribución puede interpretarse como un «salario sombra» que representa el coste de oportunidad
} 
stock de capital de mercado, $R_{t}^{s}$ la rentabilidad de los activos de capital domésticos en el sector de la economía colaborativa, y $H_{t}$ el stock de capital doméstico.

Por lo que respecta a la dotación de tiempo de las familias, podemos suponer que la asignación de tiempo se realiza entre cuatro actividades: ocio, trabajo en el mercado, tiempo dedicado a la producción de bienes y servicios en el hogar, y si el sector de la economía colaborativa requiere del mismo, tiempo asignado a la producción en la economía colaborativa. Es decir, las familias tienen que decidir cuánto tiempo van a destinar a la oferta de trabajo en el sector de mercado, cuánto tiempo van a dedicar a la producción doméstica y cuánto a la producción colaborativa, siendo el ocio un residuo del tiempo total disponible. Por tanto, la decisión del individuo respecto a la economía colaborativa se centraría en cuánto capital doméstico y cuánto tiempo van a destinar a este sector. Normalizando a la unidad la dotación inicial de tiempo tendríamos:

$$
O_{t}=1-L_{m, t}-L_{s, t}-L_{h, t}
$$

donde $L_{h, t}$ es el tiempo dedicado a la producción doméstica.

El stock de capital físico de mercado se obtendría a través de la siguiente ecuación de acumulación:

$$
K_{t+1}=\left(1-\delta_{k}\right) K_{\mathrm{t}}+I_{k, t}
$$

donde $\delta_{k} \in(0,1)$ es la tasa de depreciación física del capital.

Por su parte, el stock de capital doméstico, vendría dado por:

$$
H_{t+1}=\left(1-\delta_{h}\right) H_{\mathrm{t}}+I_{h, t}
$$

donde $\delta_{h} \in(0,1)$ es la tasa de depreciación de los bienes duraderos que conforman el stock de capital doméstico. Estos bienes duraderos o capital doméstico incluyen desde la vivienda, los vehículos, hasta los juguetes de los niños.

\section{Las empresas}

El sector productivo de la economía estaría formado por las empresas, que alquilan los factores productivos capital y trabajo a las familias. Estas empresas producirían unos bienes y servicios que denominamos de mercado, a partir de la siguiente función de producción:

$$
Y_{m, t}=F\left(L_{m, t}, K_{t}\right)
$$

del tiempo aplicado a las actividades productivas en la economía colaborativa y que podría considerarse implícito en el precio global por el servicio prestado o bien compartido en este sector. Este salario sombra será igual al salario en el mercado productivo tradicional en equilibrio. 
donde $Y_{m, t}$ es la producción de mercado de la economía. Esta producción puede destinarse tanto al consumo como a la inversión, tanto en activos de capital de mercado como en bienes duraderos que conforman el stock de capital de los hogares.

\section{La producción en el hogar}

La familia obtiene también utilidad del consumo de bienes y servicios que son producidos en el hogar, tales como lavar la ropa, limpiar, cocinar, etc. Pero también podríamos incluir servicios como los que se obtienen por el uso de la vivienda, o por la utilización de los vehículos. En este caso, se trata de bienes y servicios que no son de mercado, y para los cuales la producción es equivalente a su consumo. Esto significa que en este sector productivo no existe ahorro y la producción es directamente igual al consumo. La producción doméstica de estos bienes y servicios depende de dos factores: el capital doméstico, formado por bienes duraderos, y el tiempo dedicado a la realización de actividades productivas en el hogar. La función de producción en el hogar la podemos definir como:

$$
C_{h, t}=G\left(L_{h, t}, H_{t}\right)
$$

Por tanto, consideramos que la producción en el hogar únicamente es consumida por los miembros de dicho hogar, siendo una producción no de mercado, y que estos bienes y servicios no tienen una contraprestación monetaria, por lo que no se pueden acumular.

\section{La economía colaborativa}

El tercer sector, que es el de mayor interés para nosotros, es el correspondiente al sector de la economía colaborativa, en el cual las familias ponen a disposición de otros consumidores el stock de capital doméstico disponible. En este caso se trata de alquilar dicho stock de capital, ofreciendo unos servicios derivados del uso temporal de los mismos. Se trata de un sector que tiene unas características particulares que lo diferencian claramente de los otros dos.

Así, en primer lugar, es un sector que podemos considerar de mercado, en el sentido de que hay una prestación de servicios por parte de unas familias a otras a cambio de una contraprestación monetaria. Por tanto, se trata de un sector productivo, donde la producción no es realizada por las empresas sino por las familias.

En segundo lugar, es un sector que utiliza como factores productivos trabajo y, a diferencia del sector de producción de mercado que utiliza capital físico, capital doméstico. La idea fundamental es que el grado de utilización del capital doméstico es muy bajo. Así, los hogares propietarios de los vehículos y demás activos de capital doméstico utilizan una fracción de tiempo muy limitada, por lo que existe un exceso 
de capacidad productiva respecto a la producción doméstica. Por consiguiente, la economía colaborativa implica que los bienes duraderos que conforman el stock de capital doméstico no solo pueden usarse para la producción de bienes y servicios en el hogar, sino que pueden ponerse a disposición del mercado, dada su baja tasa de utilización.

En tercer lugar, este sector se diferencia de la economía doméstica en el hecho de que la producción se vende en el mercado y, por tanto, los ingresos que se obtienen pueden ser usados tanto para el consumo como para la inversión.

En consecuencia, este sector viene definido por una función de producción que podemos especificar, si suponemos que hace falta que las familias productoras utilicen parte de su tiempo en la producción de estos bienes y servicios, como:

$$
Y_{s, t}=\mathrm{S}\left(L_{s, t}, H_{t}\right)
$$

donde $Y_{s, t}$ es la producción de la economía colaborativa que suponemos es una función de la dotación del trabajo dedicado a esta producción y del capital doméstico.

\section{Agregación}

La consideración de la economía colaborativa supone la existencia de un sector adicional a los tradicionales que posibilita alcanzar un mayor nivel de producción y de consumo. Este hecho se deriva de poner a disposición del mercado un nivel de capital mayor, a través de la incorporación de la dotación de capital doméstico, que no solo se usa para la producción de bienes y servicios en el hogar, sino también para la producción de servicios destinados a la venta en el mercado. Si bien existe un cierto grado de sustitución de bienes y servicios producidos en el mercado por servicios producidos en el sector de economía colaborativa y pueden llegar a competir en determinados segmentos de mercado, es de esperar que la mayor cantidad de recursos productivos nos lleve a un mayor nivel de producción, por lo que el efecto agregado sería positivo.

La economía colaborativa implica la incorporación de nuevos bienes y servicios, algunos sustitutivos de otros existentes en el mercado, pero otros con nuevas características, en la cesta de consumo de las familias. El consumo total está integrado por tres tipos de bienes que son sustitutivos imperfectos, tal que:

$$
C_{t}=C\left(C_{m, t}, C_{h, t}, C_{s, t}\right)
$$

lo que supone una ampliación en las posibilidades de consumo. Por otra parte, el stock total de capital existente ahora en la economía para la realización de actividades productivas de mercado, estaría compuesto tanto por el capital físico productivo de las empresas como por el capital doméstico. Al igual que el capital doméstico tiene un grado de utilización muy bajo en la producción de bienes y servicios en el 
hogar (el hecho de que tengamos equipos-herramientas en el hogar, como un taladro, no implica que lo estemos utilizando continuamente o al menos al mismo nivel que ese mismo equipo-herramienta en una empresa), es de esperar que la economía colaborativa aumente su tasa de utilización, con la consiguiente rentabilidad asociada al mismo. Esto puede dar lugar a que las decisiones de inversión de las familias se desplacen hacia estos bienes duraderos, ya que no solo pueden ser utilizados para la producción doméstica, sino que pueden trasladarse al mercado y generar una rentabilidad adicional.

Por último, el nivel de producción total de la economía, teniendo en cuenta la producción de cada uno de los tres sectores considerados vendría dada por:

$$
Y_{t}=Y_{m, t}+C_{h, t}+Y_{s, t}
$$

\section{Regulación en la economía colaborativa}

La aparición de nuevas formas de negocio y nuevos bienes y servicios hace que en la literatura exista cierto consenso en que la economía colaborativa puede aumentar el bienestar de los consumidores de muchas formas (Koopman et al., 2015a, b; Edelman y Geradin, 2015; Muscolo y Rizzo, 2018):

- Los nuevos modelos colaborativos, a través de las plataformas en las que se sustentan, facilitan el contacto y agilizan la interacción entre un mayor número de compradores y vendedores, así como reducen las asimetrías de información mediante los mecanismos reputacionales de retroalimentación y la mayor información disponible sobre productos y servicios, lo que supone una reducción en los costes de búsqueda y de transacción.

- Por el aprovechamiento de bienes y recursos que se encuentran infrautilizados, inherente a su propia definición, la economía colaborativa representa una mejora en la eficiencia asignativa. Esta incorporación al mercado de bienes y recursos al mercado colaborativo amplía el conjunto de elección de los consumidores, en muchas ocasiones, con bienes y servicios que tienen características o condiciones que no están presentes en el mercado tradicional.

- La economía colaborativa permite que muchas personas privadas o empresas participen en el mercado como prestadores de servicios, lo que promueve mayores niveles de competencia, lo que se traduce en servicios con mejores precios y mayor calidad en comparación con los existentes en la economía productiva tradicional. Estas mejores condiciones en los precios también permiten ampliar el conjunto de bienes y servicios que, gracias a la economía colaborativa, son asequibles para los consumidores.

El reconocimiento de las mejoras en términos de bienestar que conlleva la economía colaborativa debería servir para que las autoridades competentes evitaran la 
aplicación de una regulación estricta y restrictiva de las actividades que se realizan en este ámbito. Sin embargo, existen una serie de circunstancias que motivan que la decisión adoptada no sea siempre la que seguiría de considerar únicamente esas ganancias asociadas a la economía colaborativa.

\section{¿Economía colaborativa regulada o desregulada?}

En los últimos tiempos, las medidas reguladoras introducidas parecen responder más a las exigencias planteadas por los operadores establecidos, pertenecientes a la economía productiva tradicional, que a solucionar los posibles fallos de mercado que podría haber ocasionado el resurgimiento y expansión de la economía colaborativa ${ }^{11}$. Estos operadores del sector productivo tradicional influyen o ejercen presión sobre los responsables de las políticas para que las medidas reguladoras que estos adopten limiten la competencia que ejercen los nuevos prestadores de servicios de la economía colaborativa, produciéndose el fenómeno conocido como «captura del regulador» (Dal Bó, 2006).

En ciertos casos, las regulaciones implantadas son manifiestamente proteccionistas del sector tradicional, como, por ejemplo, establecer un plazo de pre-contratación mínimo entre la contratación del servicio de coche compartido (car sharing) y la prestación efectiva del servicio, o determinados requisitos exigidos a las viviendas de uso turístico ofertadas en las plataformas colaborativas como, por ejemplo, una entrada independiente de la utilizada por los vecinos del inmueble al que pertenezcan, o también el hecho de que tenga que darse una estancia mínima para estas viviendas.

En otros casos, la introducción de medidas reguladoras a la actividad desarrollada en la economía colaborativa se ampara en la necesidad de proteger a los consumidores, por ejemplo, exigiendo que los bienes y servicios que se comparten en las plataformas cumplan una serie de requisitos. Además, se suelen alegar criterios de justicia para tratar de imponer un tratamiento regulador a los operadores de la economía colaborativa equivalente al que estarían sometidos los operadores de la economía tradicional, afectados por la competencia de los primeros, introduciendo restricciones o trabas al acceso al mercado, por ejemplo, requiriendo licencias o autorizaciones para operar, así como al ejercicio de la actividad, estableciendo límites de espacio o tiempo en el que puede desarrollarse o sobre los precios que pueden fijarse. Estas restricciones constituyen un serio obstáculo para la difusión y expansión de las ventajas que generan las nuevas innovaciones para el mercado, en particular la consecución de un mercado más competitivo.

Respecto a esta última justificación para regular la economía colaborativa, tal como señala Koopman et al. (2015b), ante las demandas legítimas de solución de

${ }^{11}$ Fundamentalmente externalidades negativas y problemas de información asimétrica, a los que hemos hecho referencia a lo largo del trabajo. 
los problemas generados por las asimetrías reguladoras entre economía tradicional y colaborativa, no parece que lo más acertado sea desalentar el desarrollo de nuevas innovaciones aplicando los regímenes reguladores antiguos a las nuevas tecnologías y sectores. La mejor alternativa consistiría en poner al mismo nivel de regulación a todos los sectores afectados reduciendo la regulación existente para el sector tradicional, en lugar de aumentar la regulación en el sector colaborativo. Salvadas las diferencias reguladoras entre ambos sectores, manteniendo solo aquellas restricciones al acceso o ejercicio de la actividad que estén justificadas por razones imperiosas de interés general (por ejemplo, salud, seguridad pública, protección del consumidor o del medio ambiente) ${ }^{12}$, los operadores del sector productivo tradicional deberían adaptar sus modelos de negocio, aprovechando el potencial que ofrecen las nuevas tecnologías, para poder competir en el mercado ${ }^{13}$.

Otra cuestión relevante que concierne al establecimiento o no de medidas reguladoras a los prestadores de servicios en la economía colaborativa, se refiere a la aplicación del principio de proporcionalidad. Sobre este particular, los prestadores de servicios que participan ocasionalmente, y no profesionalmente, en la economía colaborativa no deberían estar sometidos al cumplimiento de requisitos reguladores para el acceso o ejercicio de su actividad; y de ser necesario por razones de interés general, que estos fuesen menos estrictos que los exigidos a los prestadores profesionales de servicios. Al fin y al cabo, estos últimos simplemente estarían desarrollando distintos formatos de venta para una actividad comercial que ya ejercían, y para la que ya existe una normativa reguladora (Hatzopoulos y Roma, 2017).

La clave, entonces, es distinguir entre prestadores ocasionales y profesionales, ya que serán estos últimos los que deberán cumplir la regulación aplicable a la actividad económica de prestación de servicios. La Comisión Europea propone el establecimiento de umbrales razonables sobre determinadas variables como indicadores que ayuden a reconocer a los prestadores no profesionales o prosumidores (Comisión Europea, 2016a). En particular, plantea una combinación de variables como la frecuencia de los servicios, si existe ánimo de lucro con la actividad o el nivel de ingresos que se obtiene con la misma. En cualquier caso, independientemente de lo acertadas que serían estas variables para clasificar a los prestadores de servicios en la economía colaborativa, esta propuesta no está exenta de las pegas achacables a la discrecionalidad en el establecimiento de los umbrales en cada caso.

${ }^{12}$ Las razones imperiosas de interés general en España vienen contempladas en el artículo 3.11 de la Ley 17/2009, de 23 de noviembre, sobre el libre acceso a las actividades de servicio y su ejercicio.

13 Akbar y Tracogna (2018) clasifican las plataformas en plataformas peer-to-peer, donde una empresa desarrolla y gestiona las transacciones entre usuarios y proveedores independientes, y plataformas «integradas», en las que una empresa administra varios mecanismos incluyendo transacciones entre proveedores y usuarios independientes y pueden también poseer su propio stock de activos que se pondría a disposición de los usuarios bajo demanda. Estos autores recomiendan que las cadenas hoteleras lleven a cabo una revisión de sus modelos negocios y desarrollen plataformas «integradas» para hacer frente a los retos competitivos planteados por las plataformas de la economía colaborativa. 
Precisamente, este poder discrecional del que dispondrían las administraciones públicas puede llegar a dificultar o impedir que se alcance la unidad de mercado, situación mucho más probable cuanto más descentralizada y ausente de criterios comunes o consenso se encuentre la potestad reguladora. Diferencias en el marco regulador aplicado en distintos ámbitos (locales, nacionales o internacionales) implican que los operadores se vean sometidos a normas discriminatorias por razón del territorio en el que pretenden llevar a cabo su actividad, lo que restringe claramente el derecho básico de libertad de empresa.

En numerosas ocasiones, la aparición de la economía colaborativa ha promovido que las autoridades planteen modificaciones de la legislación existente o la introducción de nuevas normas para adaptarlas a los nuevos problemas que se presentan en este nuevo contexto. No obstante, muchos de esos problemas a los que se alude ya tendrían respuesta en la normativa existente de protección del consumidor o de defensa de la competencia. Por tanto, las autoridades competentes deberían analizar la conveniencia de introducir medidas innecesarias, dados los instrumentos que existen ya a su alcance para regular los nuevos modelos de negocio.

\section{Autorregulación en la economía colaborativa}

Una de las posibles alternativas que pueden considerarse para regular la economía colaborativa es la autorregulación. Esta consistiría en la reasignación de la responsabilidad reguladora a otras partes distintas a las autoridades públicas (Cohen y Sundararajan, 2015). En este sentido, la mayoría de las propuestas defienden el papel que deberían desempeñar las plataformas digitales colaborando de manera voluntaria u obligatoria con las autoridades (Vitkovic, 2016). De hecho, en los últimos tiempos, se observan soluciones a los posibles efectos adversos de la economía colaborativa basadas en acuerdos alcanzados entre el sector y las administraciones públicas. Un ejemplo de esta última situación es la propuesta realizada por Airbnb, fruto del acuerdo con el Ayuntamiento de París, para limitar el tiempo de alquiler de las viviendas turísticas en esa ciudad, que se ofertan en esa plataforma, con el objeto de luchar contra el fraude y reducir los efectos que esta oferta estaría teniendo sobre el mercado tradicional de alquiler de viviendas.

Aun cuando esta opción reguladora supone una opción menos distorsionadora del sector que la intervención directa de las autoridades, hay que tener presente que esta autoregulación puede llegar a pervertirse, de manera que los mecanismos reguladores creados sirvan a fines distintos a los que motivaron su implantación, perjudicando a operadores y/o usuarios. De ahí que sea necesario una revisión continua de esos sistemas reguladores para que sean los más adecuados, transparentes y eficaces en la protección del consumidor y la prevención del fraude (Stemler, 2017). 


\section{Economía colaborativa y economía sumergida}

Otro de los problemas objeto de preocupación que ha llevado aparejada la economía colaborativa es el crecimiento de la economía sumergida, incluyendo todas aquellas actividades retribuidas que no se declaran a las autoridades públicas a efectos fiscales, de la seguridad social o de la legislación laboral. Piénsese que cuando la principal o única fuente de rentas proviene de la economía colaborativa, esto se traduce en la existencia de empleo o autoempleo en el que los trabajadores carecerían de la cobertura de la seguridad social. Lógicamente esta situación resulta más preocupante en aquellas actividades donde la economía colaborativa ha alcanzado un mayor éxito, como son aquellas relacionadas con el sector del turismo o del transporte.

Los operadores pertenecientes al mercado productivo tradicional consideran competencia desleal los servicios ofertados en las plataformas no solo por no estar sometidos a la misma regulación, sino también porque muchos de ellos encuentran en estas plataformas una forma de eludir la normativa y sus obligaciones fiscales, lo que los coloca en una situación de ventaja al tener que soportar menos cargas.

Williams y Horodnic (2017) señalan que para hacer frente a la expansión de la economía sumergida en el sector de la hostelería las autoridades deberían aplicar políticas que combinaran tanto controles directos como indirectos.

En el caso de los controles directos, el acceso de las autoridades a la información recabada por las plataformas sobre los ingresos obtenidos en las transacciones que allí se realizan permitiría determinar qué actividades habrían sido declaradas y cuáles no, imponiendo sanciones que superen los beneficios de pertenecer a la economía sumergida. Además, si las plataformas advirtieran a los usuarios del acceso a esta información por parte de las Administraciones Públicas, se produciría un efecto disuasorio para estas transacciones no declaradas ${ }^{14}$. Estas medidas se podrían complementar con otras que hicieran más atractivo a los prestadores de servicios operar en el sector formal de la economía.

Por otro lado, los controles indirectos tendrían como objetivo conseguir modificar la percepción que los usuarios tienen de los participantes en la economía sumergida, de manera que, a través de una mayor información sobre las obligaciones de todas las partes y consecuencias de su incumplimiento, se logre una mayor concienciación y sean los propios agentes los que reduzcan este sector mediante una auto-regulación.

En todo caso, las consideraciones previas sobre la necesidad de tener presente el principio de proporcionalidad a la hora de imponer obligaciones (o sanciones en caso de incumplimiento) respecto a los ingresos obtenidos a través de transacciones reali-

${ }^{14}$ En España, por ejemplo, el Real Decreto 1070/2017, de 29 de diciembre, por el que se modifican el Reglamento General de las actuaciones y los procedimientos de gestión e inspección tributaria y de desarrollo de las normas comunes de los procedimientos de aplicación de los tributos, aprobado por el Real Decreto 1065/2007, de 27 de julio, y el Real Decreto 1676/2009, de 13 de noviembre, por el que se regula el Consejo para la Defensa del Contribuyente, introduce como novedad la obligación de información específica para las personas o entidades, en particular, las denominadas «plataformas colaborativas», que intermedien en la cesión del uso de viviendas con fines turísticos, con el objetivo de prevenir el fraude fiscal. 
zadas en el marco de la economía colaborativa no puede obviarse. Concretamente, no deberían tener el mismo tratamiento aquellos prestadores de servicios que participan de forma ocasional, y aquellos otros que lo hacen profesionalmente con una actividad continua o habitual. El problema, tal y como hemos analizado previamente, es establecer las líneas divisorias que distingan entre prestadores ocasionales y profesionales.

\section{Conclusiones}

En los últimos años se ha producido un desarrollo de lo que hemos venido a denominar economía colaborativa, impulsada fundamentalmente por la aparición de Internet y el desarrollo de aplicaciones que permiten conectar de forma directa a consumidores oferentes y demandantes de determinados bienes y servicios. La economía colaborativa no es un fenómeno nuevo, sino que siempre ha existido, e incluso puede llegar a constituir el principal segmento del mercado en economías poco desarrolladas. En etapas de desarrollo económico primitivo, la economía colaborativa ha sido una de las principales actividades económicas. La revolución industrial dio lugar a un desarrollo de la economía de mercado en la que la oferta de bienes y servicios se realiza principalmente a través de empresas, disminuyendo de forma considerable el peso de la economía colaborativa. Sin embargo, el progreso tecnológico ha permitido de nuevo un avance en la economía colaborativa, posibilitando el uso de bienes duraderos, que constituyen el stock de capital de los hogares, para la producción de servicios de mercado.

El desarrollo de la economía colaborativa no está exento de problemas, principalmente derivados de determinadas externalidades negativas que pueden generar, su competencia con determinadas actividades económicas de mercado, así como por la falta de un adecuado marco regulador.

Aunque realizar una proyección sobre el futuro resulte un tanto arriesgado, desde nuestro punto de vista la economía colaborativa va a ser un sector que va a seguir experimentando una gran expansión, incorporándose a la misma nuevos bienes y servicios de forma paulatina, proceso que irá de la mano del desarrollo tecnológico. Concretamente, aunque la vivienda sea el mayor activo, en términos de valor, que posean los hogares, muchas actividades de la economía colaborativa incluyen el uso de los automóviles, ordenadores personales, maquinaria, herramientas y, muy probablemente en el futuro, las impresoras 3-D, que supondrán el acceso de los hogares a la producción de un gran número de bienes. En este sentido, Petersen y Pearce (2017) realizan un interesante análisis sobre la implantación de las impresoras 3-D en los hogares para la producción de bienes manufacturados. De acuerdo con este estudio, el desarrollo de esta tecnología con el consiguiente abaratamiento de los costes permitirá más adelante extender la economía colaborativa al sector industrial. Así, bastaría con que una familia adquiriese una impresora 3-D y la utilizase para producir determinados productos industriales que podrían ser vendidos a otros consumidores. 
En este contexto, las autoridades competentes deben poner en marcha nuevos marcos reguladores, con objeto de minimizar el impacto negativo que estas actividades puedan tener sobre el bienestar social, pero sin suponer un obstáculo al desarrollo de estas nuevas actividades económicas. Así, este marco regulador tiene que adaptarse a las nuevas realidades, no siendo válidos, en la mayoría de los casos, los marcos reguladores existentes en la actualidad.

Los bienes y servicios que se comercian dentro de la economía colaborativa son productos diferenciados, aunque altamente sustitutivos, respecto a bienes y servicios similares producidos por el mercado, aumentando su variedad conforme avanza la tecnología. En este sentido, las autoridades deben adoptar una nueva perspectiva, adaptándose a esta nueva realidad, con el objeto de integrar a la economía colaborativa dentro de la estructura productiva de la economía, eliminando o al menos mitigando los problemas que puedan generarse en su proceso de desarrollo, con el objetivo final de aumentar el bienestar social.

\section{Referencias bibliográficas}

Akbar, Y. H., \& Tracogna, A. (2018). The sharing economy and the future of the hotel industry: Transaction cost theory and platform economics. International Journal of Hospitality Management, 71, 91-101.

Altinay, L., \& Taheri, B. (2018). Emerging themes and theories in the sharing economy: a critical note for hospitality and tourism. International Journal of Contemporary Hospitality Management. https://doi.org/10.1108/IJCHM-02-2018-0171.

Bardhi, F., \& Eckhardt, G. M. (2012). Access-based consumption: The case of car sharing. Journal of Consumer Research, 39(4), 881-898.

Bates, J., \& Leibling, D. (2012). Spaced Out. Perspectives on parking policy. London: Royal Automobile Club Foundation.

Becker, G.S. (1965). A theory of allocation of time. Economic Journal, 75(299), 493-517.

Belk, R.W. (1988). Possessions and the extended self. Journal of Consumer Research, 15(2), 139-168.

Belk, R. (2014). You are what you can access: Sharing and collaborative consumption online. Journal of Business Research, 67(8), 1595-1600.

Benhabid, J., Rogerson, R., \& Wright, R. (1991). Homework in Macroeconomics: Household production and aggregate fluctuations. Journal of Political Economy, 99(6), 1166-1187.

Benkler, Y. (2004). Sharing nicely: On shareable goods and the emergence of sharing as a modality of economic production. Yale Law Journal, 114(2), 273-358.

Botsman, R., \& Rogers, R. (2010a). What's Mine Is Yours: The Rise of Collaborative Consumption. London: HarperCollins.

Botsman, R. \& Rogers, R. (2010b). Beyond Zipcar: Collaborative consumption. Harvard Business Review, 88(10), 30.

Cheng, M. (2016): Sharing economy: A review and agenda for future research. International Journal of Hospitality Management, 57, 60-70.

Cohen, M., \& Sundararajan, A. (2015). Self-regulation and innovation in the peer-to-peer sharing economy. University of Chicago Law Review Dialogue, 82, 116-133. 
Comisión Europea (2016a). Una Agenda Europea para la economía colaborativa. Comunicación de la Comisión al Parlamento Europeo, al Consejo, al Comité Económico y Social Europeo y al Comité de las Regiones. COM(2016) 356 final. Bruselas, 2 de junio de 2016.

Comisión Europea (2016b). The use of collaborative platforms. Flash Eurobarometer Report 438- March 2016. Survey requested by the European Commission, Directorate-General for Internal Market, Industry, Entrepreneurship and SMEs and co-ordinated by the Directorate-General for Communication.

Coyle, D., (2018). Do-it-yourself digital: the production boundary, the productivity puzzle and economic welfare. Economica. doi: 10.1111/ecca.12289.

Dal Bó, E. (2006). Regulatory capture: a review. Oxford Journal of Economic Policy, 22 (2), 203-25.

Dredge, D., \& Gyimóthy, S. (2015). The collaborative economy and tourism: Critical perspectives, questionable claims and silenced voices. Tourism Recreation Research, 40(3), 286-302.

Edelman, B. G., \& Geradin, D. (2015). Efficiencies and regulatory shortcuts: How should we regulate companies like Airbnb and Uber. Stanford Technology Law Review, 19, 293-328.

Einav, L., Farronato, C., \& Levin, J. (2016): Peer-to-peer markets. Annual Review Economics, 8, 615-635.

Evans, D., \& Schmalensee, R. (2015). The Antitrust Analysis of Multisided Platform Businesses. The Oxford Handbook of International Antitrust Economics, 1 (pp. 404-447). London: Oxford University Press.

Frenken, K., \& Schor, J. (2017). Putting the sharing economy into perspective. Environmental Innovation and Societal Transitions, 23(1), 3-10.

Gal-Or, E. (2018). Peer-to-peer sharing in the lodging market: Evaluating implications for social welfare and profitability. Journal of Economics \& Management Strategy, 27(4), 686-704.

Greenwood, J. \& Hercowitz, Z. (1991). The allocation of capital and time over the business cycle. Journal of Political Economy, 99(6), 1188-1214.

Greenwood, J., Seshadri, A., \& Yorukoglu, M. (2005). Engines of liberation. Review of Economic Studies, 72, 109-133.

Gronau, R. (1973a). The intrafamily allocation of time: The value of the housewives' time. American Economic Review, 63(4), 634-651.

Gronau, R. (1973b). The effect of children on the housewife's value of time. Journal of Political Economy, 81(2), S168-S199, Part II.

Hatzopoulos, V., \& Roma, S. (2017). Caring for sharing? The collaborative economy under EU law. Common Market Law Review, 54(1), 81-127.

Koopman, C., Mitchell, M., \& Thierer, A. (2015a). The sharing economy and consumer protection regulation: The case for policy change. Journal of Business, Entrepreneurship \& the Law, 8 (2), 529-545.

Koopman, C., Mitchell, M.D., \& Thierer, A. D. (2015b). The sharing economy: Issues facing plat-forms, participants, and regulators. George Mason University: Mercatus Center Working Paper.

Mcgrattan, E., Rogerson, R., \& Wright, R. (1997). An equilibrium model of the business cycle with household production and fiscal policy. International Economic Review, 38(2), 267-290. 
Muscolo, G., \& Rizzo, A. M. (2018). Sharing Economy: a Multifaceted Phenomenon. Rivista Italiana di Antitrust/Italian Antitrust Review, 5(1), 95-111.

Petersen, E.E., \& Pearce, J. (2017). Emergence of home manufacturing in the developed world: Return on investment for open-source 3-D printers. Technologies, 5(7), 1-15.

Rochet, J. C., \& Tirole, J. (2006). Two-sided markets: a progress report. The RAND journal of economics, 37(3), 645-667.

Stemler, A. (2017). Feedback loop failure: Implications for the self-regulation of the sharing economy. Minnesota Journal of Law, Science \& Technology, 18, 673-712.

Sutherland, W., \& Jarrahi, M.H. (2018). The sharing economy and digital platforms: A review and research agenda. International Journal of Information Management, 43, 328-341.

Thierer, A., Koopman, C., Hobson, A., \& Kuiper, C. (2016). How the Internet, the Sharing Economy, and Reputational Feedback Mechanisms Solve the «Lemons Problem». University of Miami Law Review, 70, 830-878.

Vaughan, R., \& Daverio, R. (2016). Assessing the size and presence of the collaborative economy in Europe. PwC UK, impulse paper for the European Commission.

Vitkovic, D. (2016). The sharing economy: regulation and the EU competition law. Global Antitrust Review, 9, 78-118.

Weber, T. (2016). Product pricing in peer-to-peer economy. Journal of Management Information Systems, 33(2), 573-596.

Williams, C. C., \& Horodnic, I. A. (2017). Regulating the sharing economy to prevent the growth of the informal sector in the hospitality industry. International Journal of Contemporary Hospitality Management, 29(9), 2261-2278.

World Tourism Organization (2017). New Platform Tourism Services (or the so-called Sharing Economy) - Understand, Rethink and Adapt. Madrid, UNWTO. 